\title{
Uso de convergência tecnológica sem regulamentação apropriada: VOIP e competitividade
}

\author{
Emílio José Montero Arruda Filho \\ $\mathrm{PhD}$ Candidate in Marketing and E-commerce at University of Bergamo (UNIBG - 2005/2008)Italy. \\ E-mail: earruda@prof.iesam-pa.edu.br
}

\author{
Ruby Roy Dholakia \\ Professor of Marketing/Director of RITIM (Research Institute for \\ Telecommunications and Information Marketing College of Business Administration \\ E-mail: ruby@uri.edu
}

\section{Resumo}

A convergência tecnológica criou a possibilidade de serviços de comunicações serem realizados baseados em tecnologias diferentes, garantindo o mesmo uso para usuários e melhores benefícios para provedores. Desta forma, ambos os consumidores - empresariais e residenciais - tiveram aumentadas suas escolhas para serviços conjuntos, bem como para provedores de serviços. A regulação existente não analisa o serviço como um resultado, mas como um produto ou tecnologia a ser autorizada. Baseado na literatura de utilização e comportamento do consumidor, este artigo descreve e discute uma estratégia de marketing usada pelos operadores de telecomunicações, os quais tiram vantagem da ineficiência da regulação e legislação. Uma análise teórica do ambiente competitivo e do uso da tecnologia conclui que benefícios econômicos da convergência tecnológica ocorrem devido a quatro fatores: progresso tecnológico; falhas na regulação; complexidade da tecnologia; não habilidade de explorar os benefícios da convergência. O mau uso destes serviços pelos provedores de telecomunicações, tendo em vista a convergência tecnológica e integração de sistemas neste setor, são discutidos especificamente no contexto dos serviços de comunicação de voz à longa distância utilizando VOIP, criando propostas para definir o melhor uso da tecnologia a fim de garantir uma competição justa para o mercado.

\section{Palavras-Chave}

Convergência tecnológica. Integração de sistemas. Regulação. Competição justa. Complexidade tecnológica.

\author{
Technology convergence usage without an \\ appropriate regulation: VOIP and \\ competition
}

\begin{abstract}
Technological convergence allowed for the carrying out of communication services, based on different technologies, assuring the same use for users and better benefits for providers. This way, both entrepreneurial and residential consumers had an increase in their choice for joint services as well as for service providers. The existing regulation does not analyze the service as a result, but as a product or technology to be authorized. Based on the literature of utilization and behavior of the consumer, this article describes and discusses a strategy of marketing used by communication opoerators, who take advantage from the inefficiency of the regulation and legislation. A theoritical analysis of the competitive environment and use of technology points out that the economic benefits from technological convergence go on due to four factors: technological progress, regulation failures, technological complexity, and lack of skill for exploring convergence benefits. The misuse of these services by telecommunication providers, given the technological convergence and integration of systems in this sector, are specifically discussed in the context of voice communication services at a long distance, utilizing VOIP, establishing proposals for defining the better use of technology which may assure a fair competitiveness for the market.
\end{abstract}

\section{Keywords}

Technology convergence. System integration. Regulation. Fair competitiveness. Technology complexity. 


\section{INTRODUÇÃO}

A possibilidade de oferecer um conjunto de serviços integrados, como televisão, internet e telefonia, via uma só conexão ao consumidor (NUNES; WILSON; KAMBIL, 2000) é algo bastante atual no mercado de telecomunições. Muitas empresas americanas e européias se tornaram integradoras (SHELANSKY, 2007) capazes de oferecer este tipo de serviço, e a comunicação de voz, devido à convergência tecnológica - TC (XAVIER; YPSILANTI, 2007), é oferecida na forma de telefonia fixa, móvel e voz sobre protocolo de internet (VOIP). Com todas estas possibilidades, torna-se possível comunicarse de qualquer parte do mundo com qualquer pessoa, por meio de diferentes tipos de tecnologias conjuntamente (HAMERIA; PAATELA, 2005).

No corrente mercado de telecomunicações, dinâmico e agressivo, competidores diferentes encaram um campo de batalha desigual, onde provedores de telecomunicações com altos investimentos em infraestrutura física são capazes de tirar vantagem das estruturas de regulação maximizando sua receita e criando uma competição injusta entre empresas de comunicação de voz (fixa ou móvel) e internet. O VOIP, visto como um serviço de valor agregado e não um serviço de comunicação de voz (COHEN; MATTILA; SOUTHWOOD, 2005), permite a provedores de telecom incluir em seus custos de infraestrutura $\mathrm{O}$ desenvolvimento (JUSSAWALLA, 1999; PATHAK, 2005) e o uso de tecnologias, para reduzir custos sem transferir nenhuma economia destes para os consumidores. Em outra visão, empresas de internet não estão ligadas com as definições das regulações de telecomunicações, tornando-se prontas para tirar vantagem dos competidores, que possuem seus custos de comunicação de vOz e preços determinados. Para garantir um ambiente competitivo justo neste setor, forte política reguladora deveria ser desenvolvida para serviços de VOIP, porque, na rede IP, voz e tráfico de dados são similares em suas transmissões (MAEDA; AMARB; GIBSONB, 2006).
No futuro, regulações deverão definir os progressos tecnológicos, fornecendo benefícios públicos, bem como evitando falhas de mercado. Com base nestas falhas e objetivando seu desenvolvimento, o foco principal deste artigo é apresentar o argumento de que não é suficiente atender a um mínimo de especificações relacionadas à qualidade e competitividade no setor de telecomunicações, ou seja, é particularmente relevante para as autoridades de regulação que negociam com telecomunicações compreender a complexidade tecnológica neste mercado, sabendo que ela é alta e dinâmica, diferentemente do setor de transporte, por exemplo, no qual mudanças tecnológicas são muito mais lentas.

De forma bem clara e lógica, seria como se um consumidor presente no Rio de Janeiro usasse seu telefone celular para realizar uma chamada, enquanto viaja para Curitiba, por exemplo. A chamada poderia ser conectada por diferentes caminhos alternativos, e, se o provedor de serviço móvel utilizasse VOIP, para realizar a conexão entre os estados, utilizando-se para isto da própria rede interna (intranet entre suas filiais), este serviço não viria a ser controlado de nenhum regulador e o provedor seria capaz de tirar vantagem da nova integração, reduzindo seus custos sem nenhuma transferência ou nenhuma vantagem para o consumidor (DE BOER; EVANS, 1996; CARPENTER;LAPUERTA, 1999).

Em contrapartida, se a conexão telefônica requer muitas redes diferentes (ex. rede fixa e móvel, bem como diferentes provedores de serviços), vários reguladores deveriam ter jurisdição sobre assuntos como custos, qualidade, uso, taxas, assinaturas e outros. Enquanto o VOIP estiver sendo usado como uma ferramenta para realizar conexões entre PCs, isto deveria continuar sendo visto como um valor agregado, já que está sendo realizado entre empresas filiais ou entre a rede de internet. Porém, quando isto é realizado entre a rede de um provedor (internet de longa distância) e, em seguida, comercializado para terceiros, a tecnologia está desenvolvendo novos resultados, reduzindo custos e vendendo serviços não autorizados ou regularizados no mercado.

Ci. Inf., Brasília, v. 38, n. 1, p. 45-56, jan./abr. 2009 
No exemplo citado, a competitividade se torna injusta, porque tecnologicamente a compressão de dados digitais, aplicada ao PSK (Phase Shift Keying), que serve para transportar a comunicação de voz comum à longa distância, é inferior à compressão do VOIP, além de possuir regulação, o que não ocorre com o VOIP. Baseado na melhor qualidade de transmissão atual, pode-se transmitir cerca de 120 canais de voz sobre um canal $2 \mathrm{MB}$ de transmissão de dados (FENNEL Jr; GOBIOFF, 1983). Já no mesmo link de 2 MB, o VOIP, que converte o pacote de voz em dados (KORZENIOWSKI, 2001), pode transmitir pelo menos 240 canais de voz simultâneos. Verifica-se assim que, utilizando outra tecnologia melhor e ainda não regularizada, pode-se transmitir o dobro do número de comunicações de voz que no sistema de voz convencional autorizado e regularizado.

Como o VOIP não é regularizado, não existe maneira de departamentos de regularização saberem qual protocolo está sendo utilizado pelos provedores (LIU et al., 2005). Isto deixa a possibilidade de, para longas distâncias, qualquer operador que tenha fibra óptica, cabeamento próprio, satélite ou alguma banda de comunicação alugada possa duplicar suas chamadas ou reduzir esta banda previamente adquirida, afetando positivamente seus custos e receita. Consumidores, bem como reguladores, veem utilidade pública sem nenhuma conexão entre custos e taxas com o uso da tecnologia (TREBING, 2004; SAPPINGTON, 2005), o que na verdade deveria ser algo bem avaliado pela sua importância.

A convergência entre os serviços e os competidores de cada serviço faz com que provedores de telecom reduzam custos sem nenhuma passagem de redução de valores para os consumidores ou para conhecimento dos reguladores sobre a redução e novo uso. Para defender essa proposição, usaramse diversas perspectivas que suportaram toda a intuição apresentada, dados os problemas de regulação criados pela convergência nos serviços de telecomunicações. O primeiro, Integração de Sistemas (Systems Integration - SI), refere-se à integração de diferentes tecnologias capazes de desenvolver serviços de dois ou mais produtos (HOBDAY; DAVIES; PRENCIPE, 2005). Por exemplo, quando existe um equipamento $3 \mathrm{em} 1$ que realiza o mesmo que três equipamentos individuais sendo um produto único (ex: máquina de cópias, fax e scanner em um só).

Outro fator seria a convergência tecnológica (technology convergence - TC), que se refere à convergência entre diferentes serviços ou a convergência entre diferentes rotas (PATHAK, 2005), como a comunicação sobre uma rede fixa que envolve dois ou mais provedores diferentes. Por exemplo, quando se realiza uma chamada para um número fixo em outro país, utilizando um telefone celular e usando a comunicação por meio de um número 800, que usará VOIP para realizar a conexão de longa distância entre os dois países. O tipo de chamada (local, interurbana, internacional, móvel e outras) terá os preços diferenciados conforme o horário e o tempo de comunicação de cada operador.

A grande quantidade de SI e TC no mercado (LEE, 2007) guia para uma falha de claro entendimento sobre o uso da tecnologia pelos provedores de telecom, além da falha da regulação pelas autoridades competentes, as quais atualmente avaliam cada serviço como um produto individualizado.

O artigo é dividido nas seguintes partes: primeiro, apresenta-se uma visão geral da rede no setor de telecomunicações, sombreando as fronteiras competitivas entre tecnologias, provedores de serviços e aplicações do uso; segundo, regulações existentes e preços de serviços conjuntos são brevemente revistos; terceiro, a análise teórica do ambiente competitivo é apresentada em termos dos quatro fatores já citados: progresso tecnológico, falhas na regulação, complexidade da tecnologia e a não habilidade de explorar os benefícios da convergência. As consequências destes fatores são apresentadas no contexto de serviços de VOIP. Finaliza-se com as implicações para a reforma 
reguladora e o impacto sobre o comportamento competitivo no mercado.

\section{TECNOLOGIA DE COMUNICAÇÃO INTEGRADA}

\section{Rede fixa e tecnologia envolvida}

Os provedores de telefonia fixa têm a responsabilidade de prover comunicação de voz e dados em áreas metropolitanas, além de comunicações de média e longa distância entre estados, países e/ou continentes (BRUNEKREEFT; GROSS, 2000). Estas comunicações de redes cruzadas autorizadas são regulamentadas pelos departamentos locais e estaduais nos países de origem, ou no departamento regulador internacional no qual se efetua a ligação.

Para conectar as chamadas de longa distância, a infraestrutura entre os usuários em diferentes estados ou países tem de ser via cabo fixo, via rádio ponto a ponto ou via satélite. Em alguns países a infraestrutura melhorou, quando o cabeamento foi trocado por fibra óptica (COX et al., 1993). A diferença de tecnologias utilizadas não foi percebida pelo cliente ou por nenhum departamento regulador, porque a mudança ocorreu nos protocolos de comunicação, achados no interior dos cabos metálicos, fibra, rádio ou satélite. Para o usuário, o telefone convencional continua o mesmo, porque nada mudou em relação ao protocolo do cliente, mas entre as conexões de longa distância efetuadas entre os operadores.

Em outras palavras, a mudança aconteceu quando a comunicação deixou a central telefônica de um provedor, de um específico estado, indo para outro estado e entrando na rede interna da empresa (intranet). A figura 1 apresenta o que precisa ser modificado provendo a interconexão sem percepção de nenhuma mudança pelo usuário ou governo.

\section{FIGURA 1}

Conexão interestadual ou internacional usando o sistema convencional de voz e os pontos convergentes para o uso da nova tecnologia

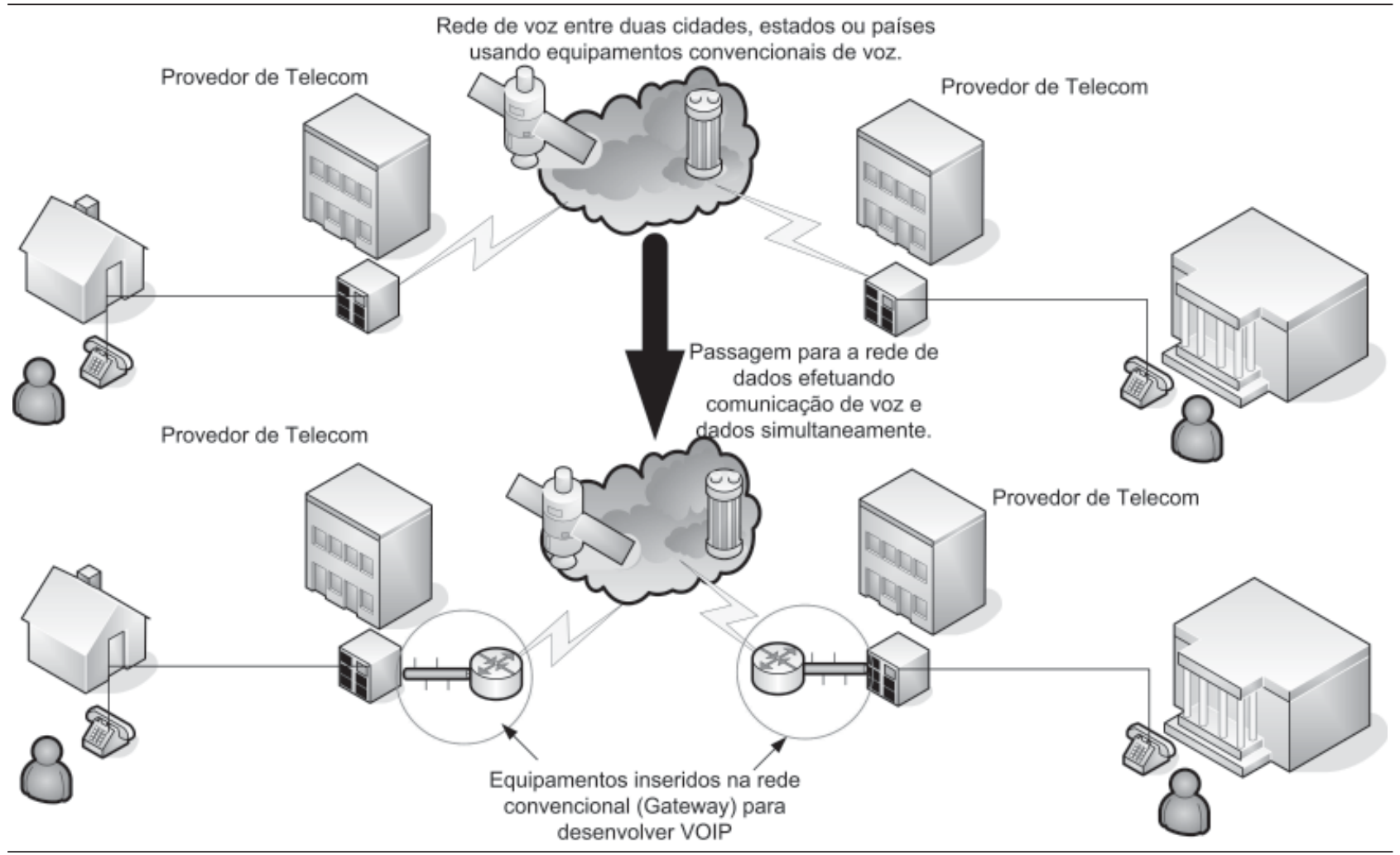


As mudanças são realizadas somente nos equipamentos de transmissão e recepção da empresa (gateways). Toda a infraestrutura de cabos, repetidores, satélites ou outros não precisam mudar, apenas o equipamento para modificar o protocolo de envio da mensagem, ou seja, o formato de comunicação desta. Os equipamentos inseridos convertem o sinal analógico de vOz em digital para protocolo de internet (CHEN; CHEN; CHIAN, 2007). Os pontos circulados na figura mostram as mudanças feitas para inserir o novo equipamento, permitindo o uso do protocolo VOIP. Este também permite compartilhar o mesmo canal de comunicação com voz, dados e imagem, com pouca deterioração da qualidade de serviço, para garantir a prioridade da transmissão (CHEN; CHEN; CHIAN, 2007). A qualidade de serviço é um termo técnico usado para garantir banda e definir as prioridades de comunicação, já que as prioridades de internet não são diretamente comunicação de voz.

\section{Rede móvel e integração}

Quando iniciado o sistema celular, ele desenvolvia comunicação utilizando suas torres celulares, que garantiam a interligação dentro de sua célula (área de abrangência conforme o projeto de cada antena) e permitiam a passagem da comunicação de uma célula para outra, enquanto o usuário falava sem existir perda da chamada. Para as ligações integradas à longa distância, era necessário utilizar parcerias com as empresas de telefonia fixa, para que elas desenvolvessem a parte interurbana ou internacional de acordo com a infraestrutura existente (BURSTEIN, 2005). Como a comunicação se expandiu, incluindo números fixos ou móveis em outras cidades, estados e até mesmo países provedores expandiram seus negócios, compartilhando e comprando serviços de seus concorrentes fixos para longas distâncias (figura 2).

\section{FIGURA 2}

Conexão Inter-estadual ou internacional usando o sistema convencional de voz e compartilhando conectividade entre os provedores móveis e fixos e depois totalmente autônomo para a rede móvel

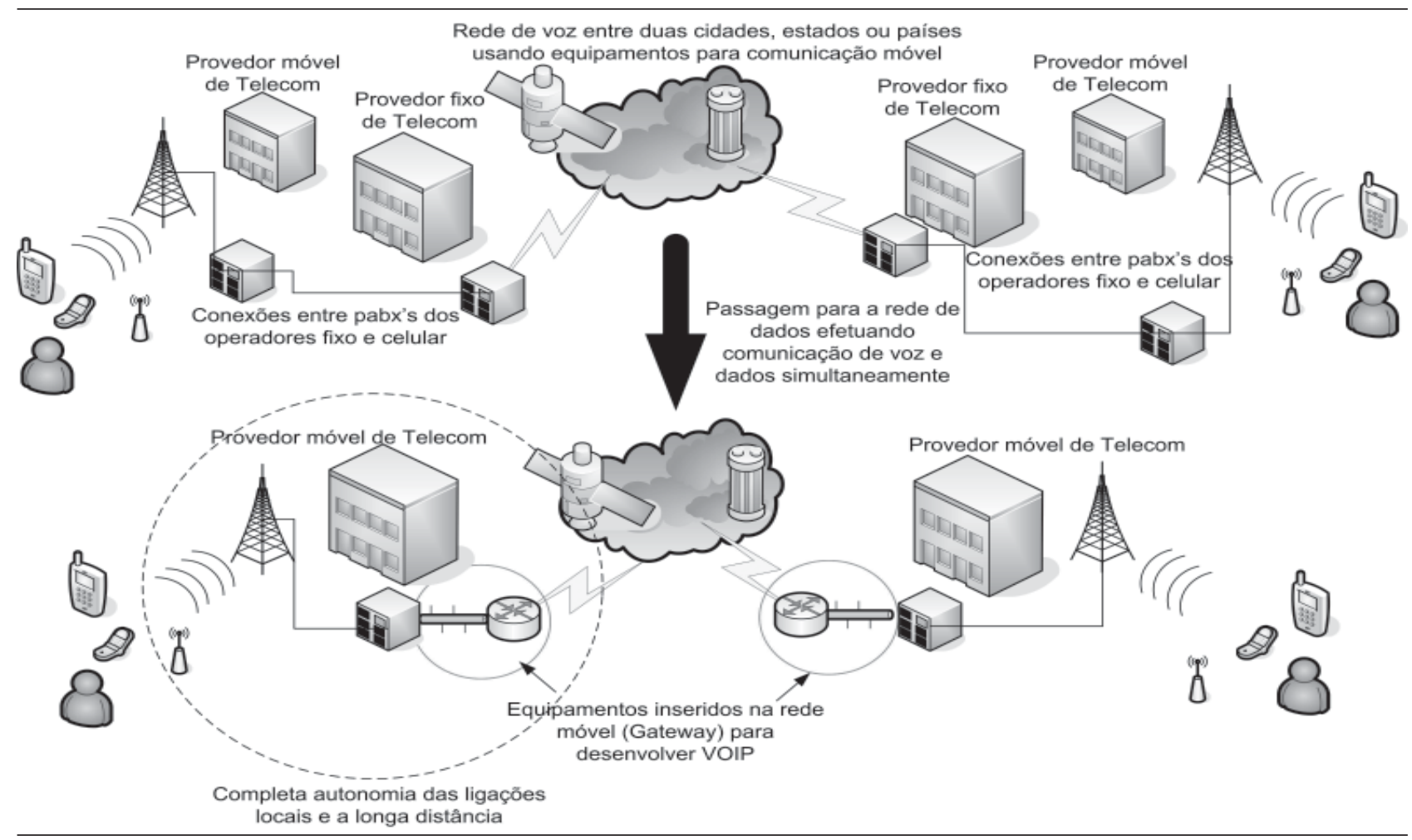


A primeira parte da figura 2 apresenta o modelo de comunicação móvel anterior ao atual, com compartilhamento da rede com o operador fixo para longas distâncias. A segunda parte da figura, abaixo da seta central, mostra a integração da voz sobre IP, além da redução do compartilhamento da rede móvel com a fixa, pois a rede móvel agora já está suficientemente autônoma. A figura 2 apenas esclarece que tanto a rede fixa quanto a móvel não obtiveram outra mudança da infraestrutura física, que não fosse a inclusão de conversores VOIP nas pontas das comunicações. A diferença é que a rede móvel otimizou ainda mais seus serviços, participando de nova fatia do mercado, desenvolvendo agora na totalidade a comunicação de um ponto a outro, mesmo a longas distâncias.

A rede móvel obteve autonomia expandindo sua infraestrutura subterrânea ou satélite de interconectividade (CHOU; LIU, 2006). Com estas implementações, o provedor móvel ganhou total conectividade, exceto para raros pontos de acesso, os quais não justificaram serem desenvolvidos pelo seu baixo uso de comunicação compartilhada. Com os compartilhamentos entre conexões quase eliminados e capazes de completar uma chamada totalmente autônoma (BLUMBERG; LUKE; CYNAMON, 2006), as empresas móveis também inseriram os dados tecnológicos, os quais reduziram bastante a comunicação de voz para longa distância. Em respeito a isto, as companhias móveis investiram diretamente na tecnologia VOIP desde o início de suas passagens autônomas, garantindo comunicações de longa distância entre seus consumidores, usando a própria infraestrutura, além de reduzir a necessidade de compartilhar seus clientes com a rede fixa.

\section{Nicho de mercado em telecom}

Em grandes países como no Brasil ou Estados Unidos, custos de desenvolvimento de infraestrutura são altos. No passado, empresas pioneiras no setor foram capazes, com suporte do estado, de otimizar suas anteriores estruturas para os atuais provedores (CRANDALL, 1997).
As empresas maiores se beneficiaram com relação as demais (DAVIES; HARDT; KELLY, 2004), porque foram capazes de compartilhar suas bandas ou seus horários de usos com outras empresas. Algumas empresas fixas e móveis que expandiram suas infraestruturas para prover serviços de longa distância a grande número de consumidores aumentaram seus negócios vendendo bandas ou alugando seu uso (GREEN, 1999).

Empresas pequenas ou empresas com alcance limitado adotaram como solução alugar o uso da rede das empresas maiores. Com links dedicados alugados e usando conexões via fibra óptica ou satélite, a ênfase principal foi implementar o protocolo, bem como os equipamentos de fronteira citados. Apesar de as comunicações enviadas terem de ser convertidas e desconvertidas em suas recepções, a quantidade de passagens (hosts) ou pontos de comunicações não afetaram a velocidade e a qualidade da comunicação provida (HABIB; CHUANG, 2007). As empresas operaram com conexões VOIP para chamadas internacionais entre empresas fixas de telefonia, e desenvolveram o que chamam de call center de voz internacional (KLUNDERT et al., 2005), nicho de chamadas internacionais a baixo preço.

Por exemplo, usando um cartão internacional, o usuário chama um número grátis 0800 (no Brasil), escuta a mensagem de um operador eletrônico explicando como desenvolver a chamada, disca o número do outro país que deseja falar, e a transferência de chamada é realizada como uma chamada local. Rapidamente esta conexão possui um enlace internacional de baixo preço.

Entre os países, a transmissão e recepção são feitas por meio das redes de operação fixa. Os usuários estão utilizando um link de dados, com tecnologia VOIP, sem sequer saber disto. Enquanto tais fontes de serviços aumentam o número de provedores de serviços em um mercado particular, isto cria desafios regulatórios como discutidos a seguir. 


\section{MODELO DE REGULAÇÃO}

Com base na integração de sistemas citada anteriormente e nos serviços fornecidos pelos provedores de telefonia fixa e móvel, os serviços convergentes permitem transmitir um canal de voz como se fosse comunicação de dados para longa distância. Isto reduz custos devido à tecnologia aplicada, bem como às taxas economizadas. Desde que não exista regulação para o sistema VOIP, a comunicação de voz é somente avaliada quando o sistema de voz convencional é usado via rede fixa ou móvel (VOGELSANG, 2003). Os modelos desenvolvidos para regulação de Price Cap e regulação da Taxa de Retorno (ROR) não levam em conta a integração entre as diferentes tecnologias (ARRUDA FILHO; CASSIA; MARINO, 2008; LEWIS; SAPPINGTON, 1989).

Os serviços convergentes na indústria de comunicações móveis fez o cenário regulatório pior do que já se apresentava. Anteriormente, no período em que o sistema era compartilhado entre operador fixo e móvel, o departamento de regulação tinha o controle das chamadas dos números móveis, quando estas eram consideradas interurbanas. Isto porque, quando uma chamada celular era efetuada para fora da sua área local, era informada pela torre mais próxima, enviando sua localização e um código de uso, além de a torre que efetuava a chamada ser obrigada a enviar os dados para a empresa fixa, de forma a efetuar a conexão entre as duas diferentes áreas (estado ou país).

Com o sistema autônomo desenvolvido pelos operadores móveis, isto não é mais fácil de identificar, porque somente o operador móvel possui toda informação sobre a completa comunicação, podendo ocultar esta. Sem nenhum equipamento de regulação capaz de controlar todas as chamadas e protocolos usados, é difícil identificar o número que efetuou a chamada e a localização originária desta chamada, exceto pelo provedor móvel.
Tecnologias diferentes possuem diferentes regulações e em alguns casos, como nos Estados Unidos, as regulações mudam entre nível estadual e federal (CHERRY, 2007). Outro fator nos EUA e também na Europa é a integração vertical entre os operadores, porque muitos provedores de telecomunicações oferecem telefonia fixa, móvel, internet e televisão, ao mesmo tempo e em um só pacote de serviços. Estas integrações são internas para um específico provedor, o qual conecta uma chamada via diversas rotas, tirando vantagens das convergências tecnológicas (SHIN, 2005; SANTOS et al., 2008).

\section{REGULAÇÃO E COMPETITIVIDADE}

O problema criado pelo SI, TC e não regulação adequada foi gerar um campo competitivo irregular, onde especialmente empresas pequenas ficaram com desvantagem, quando foram autorizadas a oferecer somente um serviço individual. Desta forma, fica claro que empresas grandes são capazes de desenvolver tecnologias diferentes, tirando vantagem da convergência para reduzir custos. A figura 3 mostra a distribuição entre o preço e a conectividade neste mercado, apresentando o

\section{FIGURA 3}

Matriz da distância da comunicação sobre o preço, levando em conta a tecnologia

\begin{tabular}{|c|c|c|c|}
\hline \multicolumn{4}{|l|}{ Preço } \\
\hline Alto & $\begin{array}{l}\text { Telefonia } \\
\text { Móvel } \\
\text { Local }\end{array}$ & $\begin{array}{c}\text { Telefonia } \\
\text { Fixa e Móvel } \\
\text { Interurbana }\end{array}$ & $\begin{array}{c}\text { Telefonia } \\
\text { Fixa e Móvel } \\
\text { Internacional }\end{array}$ \\
\hline $\begin{array}{l}\text { Médio } \\
\text { (Justo) }\end{array}$ & $\begin{array}{c}\text { Telefonia Móvel } \\
\text { Integrada a } \\
\text { Sistema de Rádio } \\
\text { (ex. Nextel) }\end{array}$ & $\begin{array}{c}\text { Telefonia Móvel e } \\
\text { Fixa Interurbana } \\
\text { utilizando VOIP } \\
\text { regulado }\end{array}$ & $\begin{array}{c}\text { Telefonia Móvel e } \\
\text { Fixa Internacional } \\
\text { utilizando VOIP } \\
\text { regulado }\end{array}$ \\
\hline \multirow[t]{3}{*}{ Baixo } & $\begin{array}{l}\text { Telefonia } \\
\text { Fixa Local }\end{array}$ & $\begin{array}{c}\text { Operadores } \\
\text { Pequenos e } \\
\text { Competitivos de } \\
\text { Telefonia Fixa } \\
\text { Interurbana } \\
\end{array}$ & $\begin{array}{l}\text { Call Center para } \\
\text { Telefonia Fixa e } \\
\text { Móvel utilizando } \\
\text { VOIP }\end{array}$ \\
\hline & \multirow[t]{2}{*}{$\begin{array}{l}\text { Baixo } \\
\text { (Local) }\end{array}$} & $\begin{array}{c}\text { Médio } \\
\text { (Interurbano) }\end{array}$ & $\begin{array}{c}\text { Alto } \\
\text { (Internacional) }\end{array}$ \\
\hline & & Distância & \\
\hline
\end{tabular}


quanto a competição é ligada diretamente ao nível da tecnologia (GABEL; HUANG, 2008) e também que é muito difícil manter competitividade ou gerar regulação nos serviços de comunicação de vOz, tratando cada tecnologia separadamente (ZHOU, 2003; PEHA; TEWARI, 1998; KLUNDERT et al., 2005).

Uma ideia do preço justo proposto pela figura 3 seria integrar sistemas já regulados otimizando o uso, como o exemplo do Nextel apresentado na figura. Seria necessário projetar sistemas sem regulação, para serem integrados (MAH, 2005) após uma regulação justa, que repasse a redução econômica desenvolvida ou parte dela para seus consumidores. O Importante é que o justo proposto seria um valor máximo deste justo, como, por exemplo, para distância média e preço baixo da figura, a competição existente que gera preços baixos é valorosa para desenvolver este mercado. Já para distância alta e preço baixo, existe o uso errado do VOIP, com empresas de call center aproveitando-se das falhas de regulação para tirar vantagem da economia e suprir alguma falha ou necessidade de mercado.

Um exemplo de regulação e competição seria entre operadores móveis adotando a portabilidade, que seria a transferência do número celular para qualquer outro operador, permitindo que o cliente não estivesse preso na operadora pelo fato de perder seu número antigo na troca. Isto automaticamente oferece mais competição ao mercado, pois o consumidor poderia agora escolher seu provedor dada a qualidade de comunicação, o custo e outros fatores mais estratégicos.

Outro ponto importantíssimo da portabilidade é que operadores atuais fazem as promoções para as ligações efetuadas apenas para números da mesma operadora, o que dificulta a entrada de novos operadores no mercado. Qualquer empresa que já tenha muito tempo no mercado possui muitos consumidores, logo o preço que estes oferecem para compartilhar suas redes com os competidores é alto, dificultando que um novo operador tenha preços razoáveis para oferecer entre seu número e o número do outro concorrente, que possui a rede antiga com a maioria do mercado.

Desta forma, as empresas mais velhas mantêm-se com a maioria de consumidores em sua rede, pois sua rede de amizades e profissional está na mesma rede com os mesmos descontos. Com a portabilidade, não se saberá que número é de que operador, logo os provedores devem batalhar em promoções para quaisquer números, sejam de seu competidor ou não, tornando a competição mais justa para empresas pequenas e grandes, lutando agora por conexões mais baratas em geral ou com mais qualidade que justifique o preço.

Os autores acreditam que, em vez de regular uma tecnologia específica, o foco da regulação deveria ser certificar serviços que desenvolvam o mesmo resultado, não importando a tecnologia. Hoje, é possível desenvolver comunicação de vOz através da internet, o que pode ser provido por uma empresa de telefone, bem como por uma empresa de internet. Isto modificaria o foco e diminuiria o trabalho de determinação de custos e preços para serviços específicos. Embora as tecnologias tenham diferentes custos com base em suas infraestruturas e investimentos, os desafios não deveriam desencorajar as autoridades de regulação, por determinados custos de operação e desenvolvimento, nos quais se deve definir como taxar estes serviços similares, desenvolvidos por tecnologias diferentes.

Como discutido anteriormente, a atual regulação suporta as grandes empresas, as quais são capazes de tirar vantagens das convergências tecnológicas, reduzindo seus custos sem pagar os devidos impostos e taxas para o governo, além de não reduzir preços para os consumidores. Grandes empresas beneficiam-se da atual regulação focando em seus grandes alcances (MANSELL, 1997), mas isto cria um campo injusto de competição horizontal, dificultando pequenas empresas de se organizarem e competir.

Em razão desta desvantagem, projetos diferentes têm sido iniciados no mundo, gerando novas formas 
de competição. Por exemplo, na Itália, foi criado um Operador de Redes Virtuais Móveis (Mobile Virtual Network Operator - MVNO) (KIM; SEOL, 2007), o qual iniciou a vender comunicação móvel, mas sem nenhum investimento direto na infraestrutura do setor. O MVNO opera como uma nova empresa celular, com números específicos, marca própria, cartões pré-pagos, sítios (sites) de conta, acesso a crédito, dentre outros. Esta empresa usa a infraestrutura das empresas existentes no setor, buscando ofertas no mercado para realizar parceria com a empresa que oferecer os melhores termos, qualidade e preços (aluguel) dos números do telefone celular, além de uma porção do sistema de comunicação. A conectividade tecnológica é mantida pelos provedores de telecomunicações anteriores, os quais se beneficiam do aumento dos números de usuários, mesmo que isto signifique dividir seu trabalho comercial.

A figura 4 apresenta a convergência das tecnologias em diferentes serviços simultaneamente. Ilustram-se as limitações e possibilidades, como, por exemplo, quando uma televisão funciona pela conexão de internet (SANTOS et al., 2008; SHIN, 2005), ocupando outro interessante mercado (BUSKIRK; SCHMIDT; RALPH, 2007). Com a chegada do Wi-Max (MARTIKAINEN, 2006), será possível conectar-se através da internet de qualquer lugar, acessando banda larga de internet dentro do carro, no meio da rua, usando computador portátil, agenda eletrônica PDA (Personal Digital Assistant), ou outro equipamento wireless (SANMATEU et al., 2002). Um pequeno PDA com software VOIP integrado seria capaz de realizar comunicação de voz sem custos e sem taxas no setor de telecomunicações, baseado no acesso de internet provido pelo Wi-Max. Esse seria outro caso de

\section{FIGURA 4}

competição injusta, porém agora, para o setor de comunicações móveis, pois todos esses fatores deveriam ser considerados para determinar um futuro mercado competitivo de forma regulada e justa.

Os fatores descritos precisam ser avaliados cuidadosamente na ordem de promover bens públicos, bem como uma competição justa. O VOIP, por exemplo, poderia ser regulado como comunicação de voz, o qual, caso desenvolvido por dois computadores em uma rede de internet, seria grátis por ser um serviço de valor agregado. Entretanto, quando o VOIP é um complemento ou integração de outro serviço, desenvolvendo comunicação com uma tecnologia aplicada na rede intranet de uma empresa, para ser comercializado ou ainda integrado a um conversor de comunicação (gateway) com um PABX, deveria ser considerado uma comunicação de vOz integrativa, portanto elegível, para todas as taxas e respectivos custos. Para regularizar e controlar os protocolos usados
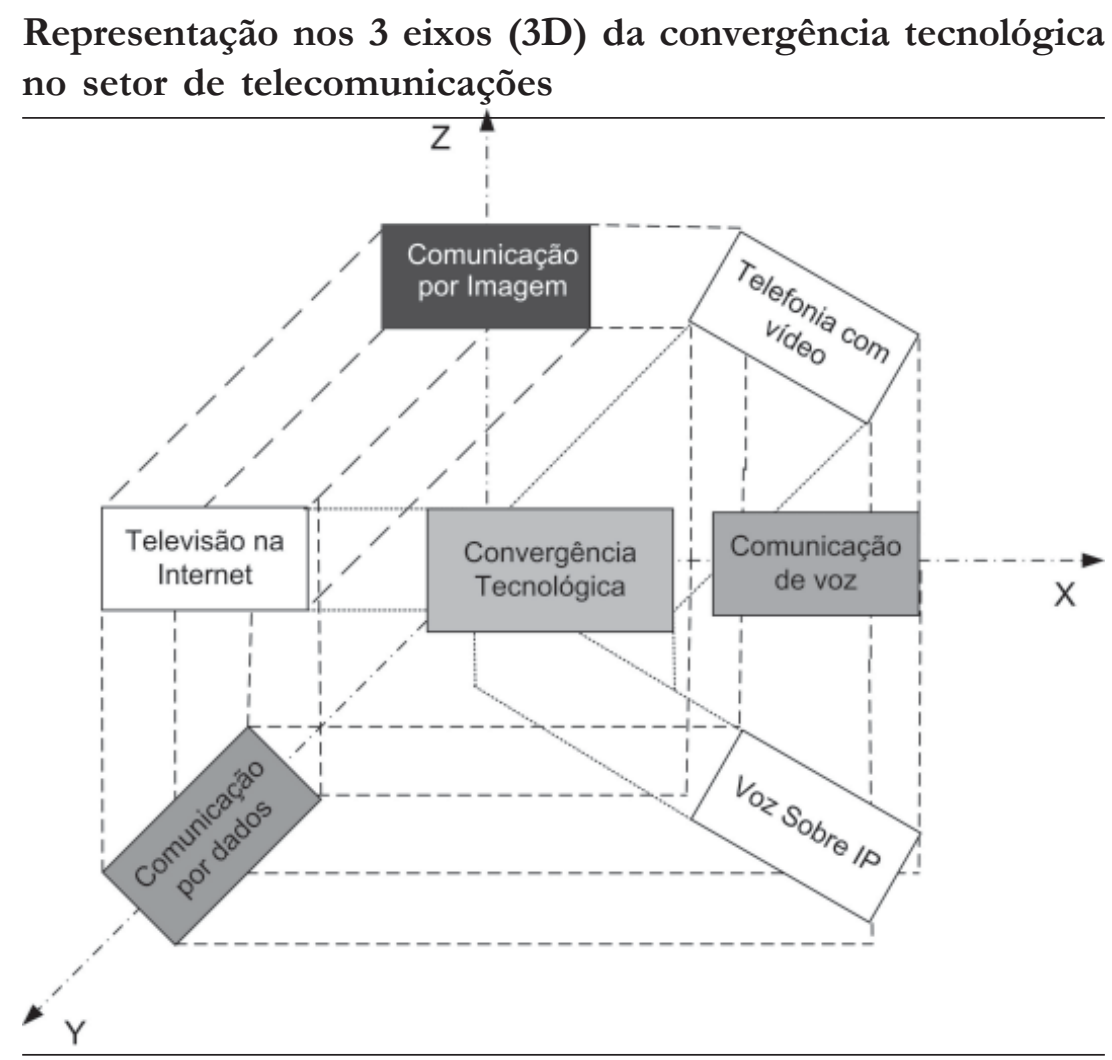
pelas companhias de telecomunicações, propõemse duas alternativas, apresentadas a seguir.

\section{Avaliação da comunicação}

Um equipamento de rede gerencial poderia ser inserido na rede dos provedores de serviço, garantindo que todas as comunicações fossem avaliadas em paralelo ao seu funcionamento. Assim, todos os protocolos relacionados com VOIP poderiam também ser definidos automaticamente. Isto poderia verificar as destinações e origens das comunicações, obrigando provedores a desenvolver desvios (proxies) que bloqueassem o tráfico com protocolo de voz na internet, não autorizado. Se o número IP final é um roteador com endereço MAC, isto automaticamente poderia ser um desvio convergente, modificado para o já citado uso na telefonia comercial. Comunicações via internet, mediante o uso de computadores ou via links dedicados sem QoS (Qualidade de serviço), deveriam ser registradas por um software livre oferecido, fornecendo uma comunicação VOIP sem custo adjunto.

A única maneira de manter e garantir a ordem seria registrando todos os softwares autorizados para realizar VOIP, fazendo um compromisso de prover o necessário para a regulação dos dados de cada chamada, quando esta não é direcionada para outro PC. Todos os sistemas usando protocolos de VOIP que não estivessem registrados ou autorizados deveriam ser bloqueados na rede internecional (CHERRY, 2005). Desta maneira, a segurança de comunicação e as regras seriam garantidas.

Dois fatores deveriam ser verificados. O primeiro é que atualmente o QoS ainda não existe na internet, que então não pode definir o serviço como sendo profissional, já que não há o mínimo de qualidade na comunicação do serviço necessário ao setor. A segunda consideração é quando o VOIP faz parte de um serviço integrativo, no qual companhias podem ter um link de dados privado, um link de dados alugado ou vendido para terceiros, o qual deveria ser controlado pelo protocolo usado. Entretanto, isto não seria permitido para ser comercializado sem que possuísse anterior autorização dos órgãos competentes, responsáveis pelo setor de telecomunicações, e também por aqueles do setor de internet.

Em geral, toda a comunicação deveria ser controlada, designando quem poderia fornecer VOIP de forma profissional e comercial. O custo ao usuário seria menor para este e ainda geraria lucros para a empresa, como proposto na figura 3, bem como garantiria uma competição mais justa para as empresas de telecomunicações, que também usam ou não a tecnologia VOIP.

\section{Definição da comunicação mais eficiente}

Outra alternativa seria regular e cobrar todos os serviços de longa distância forçando o uso do VOIP, colocando assim a comunicação convencional integrada. Um provedor sem a integração necessária para usar VOIP deveria alugar esta capacidade de um competidor, recebendo um período máximo para se atualizar de forma a ser autossuficiente. Como o VOIP é uma tecnologia que já está bastante clara no mercado, deveria ser a tecnologia utilizada por todos os provedores neste momento. Esta proposição favorece o uso de tecnologias criadas para possuir maior eficiência no sistema e passar certa parte da economia para os consumidores.

Olhando mais à frente, novas tecnologias entrarão no mercado e novos protocolos de VOIP ou outras tecnologias similares, capazes de redução de banda para transmissão, estarão disponíveis. Então, devese estar preparado para este segundo estágio da nova introdução e uso da tecnologia. Primeiro, a nova tecnologia deverá estar disponível para uso interno em empresas ou na internet, para garantir adaptação e testes no mercado. Uma vez que a nova tecnologia seja aprovada como capaz de garantir ao menos o mesmo nível de qualidade, serviço e outras necessidades tecnológicas, além de a empresa pioneira estar disponível para assumir o investimento com esta, poder-se-ia comercializar a tecnologia e seu serviço, além de repassar certa parte da economia ou redução de custos 
relacionados com o uso para o consumidor. Assim, isto aceleraria o uso da tecnologia por outros provedores de serviços. O objetivo seria encorajar empresas competidoras a usar da mesma tecnologia eficiente, tão rápido quanto possível (YUNGCHING; TSUI-HSU, 2006). Fazendo isto, motivarse-á o uso de atualizações ou tecnologias no estadoda-arte para todo provedor de serviço. Em tal caso, empresas escolhendo continuar com a obsoleta ou desatualizada tecnologia não deverão ser protegidas pelos reguladores, e claramente seus custos aplicados estariam maiores que os do competidor atualizado, perdendo automaticamente mercado nesse momento.

\section{CONCLUSÃO}

O objetivo geral deste artigo foi salientar a necessidade da regulação, garantindo um campo com competição justa entre diversos provedores de telecomunicações, além de encorajar inovações mediante o uso de novos produtos e tecnologias. O principal problema de hoje vem da complexidade da tecnologia, a qual torna difícil para reguladores e também grandes consumidores identificar e proteger o interesse de usuários, enquanto tecnologias integradas permitem produtos e serviços a serem conjugados. Regulações atuais continuam avaliando os serviços separadamente, criando regras para cada serviço como um sistema independente. Os benefícios da integração de sistemas (SI) e da convergência tecnológica (TC), em termos de baixar os custos, não são transferidos para outros usuários.

Este problema é mais severo em outras partes do mundo. Por exemplo, no Brasil, comparado com os EUA e Europa, existe pouquissíma ênfase em reduzir custos e aumentar a competição no mercado. Dada a natureza global da indústria de telecomunicações, atenções para regulações, que são mais apropriadas para o estado do desenvolvimento da tecnologia e seus impactos na competição do mercado, podem fazer grandes diferenças.

Ci. Inf., Brasília, v. 38, n. 1, p. 45-56, jan./abr. 2009
A competitividade está diretamente conectada com as oportunidades, de forma igual aos seus provedores de serviço, logo a regulação recebe importante papel no desenvolver destas oportunidades: manter um nível de uso adequado para seus consumidores.

Artigo submetido em 26/11/2008 e aceito em 08/03/2009.

\section{REFERÊNCIAS}

ARRUDA FILHO, E. J. M.; CASSIA, F.; MARINO, A. Beyond the interoperability of telephony, VoIP and networking: self-realisation marketing contribution to value creation in telecommunications sector. International Journal of Technology Marketing, v. 3, n. 1, 2008.

BLUMBERG, S. J.; LUKE, J. V.; CYNAMON, M. L. Telephone coverage and health survey estimates: evaluating the need for concern about wireless substitution. American Journal of Public Health, v. 96, n. 5, p. 926, May 2006.

BRUNEKREEFT, G.; GROSS, W. Prices for long-distance voice telephony in Germany. Telecommunications Policy, n. 24, p. 929-945, 2000 .

BURNSTEIN, D. E. An examination of market power in the intrastate long-distance telephone service markets: evidence from a natural experiment. Journal of Law and Economics, v. 48, 2005.

BUSKIRK, B.; SCHMIDT, S. M. P.; RALPH, D. L. Patterns in high-tech firms growth strategies by seeking mass mainstream customer adaptations. The Business Review, v. 8, n. 1, p. 34, 2007.

CARPENTER, P.; LAPUERTA, C. A critique of light-handed regulation: the case of British Gas February 1999. Northwestern Journal of International Law \& Business, v. 19, n. 3, p. 479, Spring 1999.

CHEN, J. L.; CHEN, M. C.; CHIAN, Yi-Ru. QoS management in heterogeneous home networks. Computer Networks, n. 51, p. 33683379, 2007.

CHERRY, S. The VoIP backlash. IEEE Spectrum, v. 42, n. 10, p. 61-63, Oct. 2005.

CHERRY, B. A. The telecommunications economy and regulation as coevolving complex adaptive systems: implications for federalism. Federal Communications Law Journal, v. 59, n. 2, p.369, Mar. 2007.

CHOU, Y.; LIU, K. C. Paradoxical impact of asymmetric regulation in Taiwan's telecommunications industry: restriction and rent seeking. Telecommunications Policy, v. 30, p. 171-182, 2006.

COHEN, T.; MATTILA, O.; SOUTHWOOD, R. VoIP and regulation, Global Symposium for regulators (GSR), Hammamet, Tunisia. Nov. 2005. Disponível em: <http://www.itu.int/osg/ spu/newslog/VoIP+And+Regulation.aspx>. Acesso em: 2009.

COX, L. A. Jr. et al. Optimal expansion of fiber-optic telecommunications networks in metropolitan areas. Interfaces, v. 23, n. 2, p. 14-35, Mar./Apr. 1993. 
CRANDALL, R. W. Are telecommunications facilities 'infrastructure?' If they are, so what?. Regional Science and Urban Economics, v. 27, p. 161-179, 1997.

DAVIES, G.; HARDT M.; KELLY, F. Come the revolution: network dimensioning, service costing and pricing in a packet switched environment. Telecommunications Policy, v. 28, p. 391-412, 2004.

DE BOER, D. B.; EVANS, L. The economic efficiency of telecommunications in a deregulated market: the case of New Zealand. Economic Record, v. 72, n. 216, p. 24, Mar. 1996.

FENNEL JR., J. W.; GOBIOFF, B. D. A satellite communications controller. IBM Systems Journal, v. 22, n. 1/2, p. 80, 1983.

GABEL, D. J.; HUANG, K. G. L. Promoting innovation and the deployment of advanced telecommunications services to businesses. Contemporary Economic Policy, v. 26, n. 2, p. 229-247, 2008.

GREEN, W. Unlocking the Potential of Outsourcing. Telecommunications, v. 33, n. 8, p. 77, Aug. 1999.

HABIB, A.; CHUANG, J. Improving application QoS with residential multihoming. Computer Networks, v. 51, p. 3323-3337, 2007.

HAMERIA, A. P.; PAATELA, A. Supply network dynamics as a source of new business. International Journal Production Economics, v. 98, p. 41-55, 2005.

HOBDAY, M.; DAVIES, A.; PRENCIPE, A. Systems Integration: a core capability of the modern corporation. Industrial and Corporate Change; v. 14, n. 6, p. 1109-1143, Nov. 2005.

JUSSAWALLA, M. The impact of ICT convergence on development in the asian region. Telecommunications Policy, v. 23, p. 217-234, 1999.

KIM, B. W.; SEOL, S. H. Economic analysis of the introduction of the MVNO system and its major implications for optimal policy decisions in Korea. Telecommunications Policy, v. 31, p. 290-304, 2007.

KLUNDERT, J. van de et al. Selecting telecommunication carriers to obtain volume discounts. Interfaces, v. 35, n. 2, p. 124, Mar./ Apr. 2005.

KORZENIOWSKI, P. VOIP-still only a drop in the bucket. Business Communications Review, v. 31, n. 2, p. 78, Feb. 2001.

LEE, G. K. The significance of network resources in the race to enter emerging product markets: the convergence of telephony communications and computer networking, 1989-2001. Strategic Management Journal, v. 28, n. 1, p. 17, 2007.

LEWIS, T. R.; SAPPINGTON, D. E. M. Regulatory option and price-cap regulation. The Rand Journal of Economics, v. 20, n.3, p. 405, Autumn 1989.

LIU, F. et al. An Approach to Integrating SIP in Converged Multimodal: multimedia communication services. Telecommunication Systems, v. 28, n. 3/4, p. 387-405, 2005.
MAEDA, T.; AMARB, A. D.; GIBSONB, A. Impact of wireless telecommunications standards and regulation on the evolution of wireless technologies and services over Internet protocol. Telecommunications Policy, v. 30, p. 587-604, 2006.

MAH, D. C. H. Explaining Internet connectivity. The Information Society, v. 21, p. 353-366, 2005.

MANSELL, R. Strategies for maintaining market power in the face of rapidly changing technologies. Journal of Economic Issues, v. 4, p. 969, Dec. 1997.

MARTIKAINEN, O. E. Complementarities creating substitutes: possible paths towards 3G, WLAN,/WiMAX and Ad Hoc Networks. The Journal of Policy, Regulation and Strategy for Telecommunications, Information and Media, v. 8, n. 4, p. 21-32, 2006.

NUNES, P.; WILSON, D.; KAMBIL, A. The all-in-one market. Harvard Business Review, Boston, v. 78, n. 3, p. 19-21, May/June 2000 .

PATHAK, J. Risk management, internal controls and organizational vulnerabilities. Managerial Auditing Journal, v. 20, n. 6, p. 569, 2005.

PEHA, J.; TEWARI, S. The results of competition between integrated-services telecommunications carriers. Information Economics and Policy, v. 10, p. 127-155, 1998.

SANMATEU, A. et al. Seamless Mobility Across IP Networks Using Mobile IP. Computer Networks, v. 40, p. 181-190, 2002.

SANTOS, J. et al. Multicast / broadcast network convergence in the next generation mobile networks. Computer Networks, v. 52, p. 228-247, 2008.

SAPPINGTON, D. E. M. Regulating service quality: a survey. Journal of Regulatory Economics; v. 27, n. 2, p. 123-154, 2005.

SHELANSKI, H. A. adjusting regulation to competition: toward a new model for U.S. telecommunications policy. Yale Journal on Regulation, v. 24, n. 1, p. 55, Winter 2007.

SHIN, D. H. Technology convergence and regulatory challenge: a case from korean digital media broadcasting. The Journal of Policy, Regulation and Strategy for Telecommunications, v. 7, n. 3, p. 47, 2005.

TREBING, H. M. Assessing deregulation: the clash between promise and reality. Journal of Economic Issues, v. 38, n. 1, p. 1, Mar. 2004.

VOGELSANG, I. Price regulation of access to telecommunications network. Journal of Economic Literature, v. 41, p. 830-862, 2003.

XAVIER, P.; YPSILANTI, D. Universal service in an IP-enabled NGN environment. The Journal of Policy, Regulation and Strategy for Telecommunications, Information and Media, v. 9, n. 1, p. 15-31, 2007.

YUNG-CHING, H.; TSUI-HSU, T. The impact of dynamic capabilities with market orientation and resource-based approaches on npdprojevt performance. Journal of American Academy of Business, v. 8 , n. 1, p. 215, Mar. 2006.

ZHOU, H. Integration and access regulations in telecommunications. Information Economics and Policy, v. 15, p. 317326, 2003. 